\title{
CPT and Lorentz Tests in Hydrogen and Antihydrogen
}

\author{
Robert Bluhm, ${ }^{1}$ V. Alan Kostelecký, ${ }^{2}$ and Neil Russell ${ }^{2}$ \\ ${ }^{1}$ Physics Department, Colby College, Waterville, Maine 04901 \\ ${ }^{2}$ Physics Department, Indiana University, Bloomington, Indiana 47405
}

(Received 20 July 1998)

\begin{abstract}
Signals for $C P T$ and Lorentz violation at the Planck scale may arise in hydrogen and antihydrogen spectroscopy. We show that certain $1 S-2 S$ and hyperfine transitions can exhibit theoretically detectable effects unsuppressed by any power of the fine-structure constant. [S0031-9007(99)08715-3]

PACS numbers: 11.30.Er, 12.20.Fv, 32.10.Fn, 32.80.Pj
\end{abstract}

Experimental and theoretical studies of the spectrum of hydrogen $(\mathrm{H})$ have historically been connected to several major advances in physics [1]. The recent production and observation of antihydrogen $(\overline{\mathrm{H}})[2,3]$ makes it plausible to consider a new class of spectroscopic measurements involving high-precision comparisons of the spectra of $\mathrm{H}$ and $\overline{\mathrm{H}}$ [4]. The two-photon $1 S-2 S$ transition has received much attention because an eventual measurement of the line center to about $1 \mathrm{mHz}$, corresponding to a resolution of one part in $10^{18}$, appears feasible [5]. It has already been measured to 3.4 parts in $10^{14}$ in a cold atomic beam of $\mathrm{H}$ [6] and to about 1 part in $10^{12}$ in trapped H [7]. Proposed $\overline{\mathrm{H}}$ spectroscopic investigations involve both beam and trapped-atom techniques $[8,9]$.

We consider here the use of spectroscopy of free or magnetically trapped $\mathrm{H}$ and $\overline{\mathrm{H}}$ to search for $C P T$ and Lorentz violation. The discrete symmetry $C P T$ is an invariance of all local Lorentz-invariant quantum field theories of point particles [10], including the standard model and quantum electrodynamics (QED). However, the situation is less clear for a more fundamental theory combining the standard model with gravity, such as string theory, where spontaneous breaking of these symmetries may occur [11]. Low-energy manifestations would be suppressed by a power of the ratio of the low-energy scale to the Planck scale, so only a few exceptionally sensitive experiments are likely to detect them.

In this paper, we show that effects of this type from the Planck scale can appear in $\mathrm{H}$ and $\overline{\mathrm{H}}$ spectra at zeroth order in the fine-structure constant. Moreover, these effects are theoretically detectable not only in $1 S-2 S$ lines but also in hyperfine transitions.

Our analysis is performed in the context of a standardmodel and QED extension [12] incorporating the idea of spontaneous $C P T$ and Lorentz breaking at a more fundamental level. This quantum field theory appears at present to be the only existing candidate for a consistent extension of the standard model based on a microscopic theory of $C P T$ and Lorentz violation. Desirable features such as energy-momentum conservation, gauge invariance, renormalizability, and microcausality are expected [12]. The theory has been applied to photon properties [12], neutralmeson experiments [11,13-15], Penning-trap tests [16], and baryogenesis [17].
We begin with a study of the spectra of free $\mathrm{H}$ and $\overline{\mathrm{H}}$. For this case, the standard-model extension generates a modified Dirac equation for a four-component electron field $\psi$ of mass $m_{e}$ and charge $q=-|e|$ in the proton Coulomb potential $A^{\mu}=(|e| / 4 \pi r, 0)$. With $i D_{\mu} \equiv i \partial_{\mu}-q A_{\mu}$, this equation (in units with $\hbar=$ $c=1)$ is

$$
\begin{gathered}
\left(i \gamma^{\mu} D_{\mu}-m_{e}-a_{\mu}^{e} \gamma^{\mu}-b_{\mu}^{e} \gamma_{5} \gamma^{\mu}\right. \\
\left.-\frac{1}{2} H_{\mu \nu}^{e} \sigma^{\mu \nu}+i c_{\mu \nu}^{e} \gamma^{\mu} D^{\nu}+i d_{\mu \nu}^{e} \gamma_{5} \gamma^{\mu} D^{\nu}\right) \psi=0 .
\end{gathered}
$$

The two terms involving the couplings $a_{\mu}^{e}$ and $b_{\mu}^{e}$ violate $C P T$, while the three terms involving $H_{\mu \nu}^{e}, c_{\mu \nu}^{e}$, and $d_{\mu \nu}^{e}$ preserve $C P T$. All five couplings break Lorentz invariance and are assumed to be small [12]. A modified Dirac equation also exists for a free proton [16], and it contains corresponding couplings $a_{\mu}^{p}, b_{\mu}^{p}, H_{\mu \nu}^{p}, c_{\mu \nu}^{p}$, and $d_{\mu \nu}^{p}[18]$.

To examine the spectra of free $\mathrm{H}$ and $\overline{\mathrm{H}}$, it suffices to perform a perturbative calculation in the context of relativistic quantum mechanics. In this approach, the unperturbed Hamiltonians and their eigenfunctions are the same for $\mathrm{H}$ and $\overline{\mathrm{H}}$. Moreover, all perturbative effects from conventional quantum electrodynamics are also identical for both systems. However, the $C P T$ - and Lorentz-breaking couplings for the electron and positron can provide different Hermitian perturbations to the Hamiltonians describing $\mathrm{H}$ and $\overline{\mathrm{H}}$. The explicit forms of these perturbations are found from Eq. (1) by a standard procedure involving charge conjugation (for $\overline{\mathrm{H}}$ ) and field redefinitions [16]. Similarly, $C P T$ - and Lorentz-breaking couplings for the proton and antiproton generate additional energy perturbations. These can be obtained to leading order using relativistic two-fermion techniques [19].

Let $J=\frac{1}{2}$ and $I=\frac{1}{2}$ denote the (uncoupled) electronic and nuclear angular momenta, respectively, with third components $m_{J}, m_{I}$. The energy corrections for the basis states $\left|m_{J}, m_{I}\right\rangle$ can then be calculated perturbatively. To leading order, we find that the energy corrections for spin eigenstates of protons or antiprotons have the same mathematical form as those for electrons or positrons, except for the replacement of superscripts $e$ with $p$ on the $C P T$ - and Lorentz-violating couplings.

C 1999 The American Physical Society 
For $\mathrm{H}$, we find that the $1 S$ and $2 S$ levels acquire identical leading-order energy shifts. They are [20]

$$
\begin{aligned}
\Delta E^{\mathrm{H}}\left(m_{J}, m_{I}\right) \approx & \left(a_{0}^{e}+a_{0}^{p}-c_{00}^{e} m_{e}-c_{00}^{p} m_{p}\right) \\
& +\left(-b_{3}^{e}+d_{30}^{e} m_{e}+H_{12}^{e}\right) m_{J} /\left|m_{J}\right| \\
& +\left(-b_{3}^{p}+d_{30}^{p} m_{p}+H_{12}^{p}\right) m_{I} /\left|m_{I}\right|,
\end{aligned}
$$

where $m_{p}$ is the proton mass. For $\overline{\mathrm{H}}$, the $1 S$ and $2 S$ levels also acquire identical leading-order energy shifts $\Delta E^{\overline{\mathrm{H}}}$, which are given by the expression (2) with the substitutions $a_{\mu}^{e} \rightarrow-a_{\mu}^{e}, d_{\mu \nu}^{e} \rightarrow-d_{\mu \nu}^{e}, H_{\mu \nu}^{e} \rightarrow-H_{\mu \nu}^{e} ; a_{\mu}^{p} \rightarrow-a_{\mu}^{p}$, $d_{\mu \nu}^{p} \rightarrow-d_{\mu \nu}^{p}, H_{\mu \nu}^{p} \rightarrow-H_{\mu \nu}^{p}$.

The hyperfine interaction couples the electron and proton or positron and antiproton spins. Denoting the total angular momentum by $F$, the appropriate basis states become linear combinations $\left|F, m_{F}\right\rangle$ of the $\left|m_{J}, m_{I}\right\rangle$ states. The selection rules for the two-photon $1 S$ - $2 S$ transition are $\Delta F=0$ and $\Delta m_{F}=0$ [21]. There are thus four allowed $1 S$-2S transitions for both $\mathrm{H}$ and $\overline{\mathrm{H}}$, occurring between states with the same spin configuration. However, according to Eq. (2), the $1 S$ and $2 S$ states with the same spin configuration have identical leading-order energy shifts, so no leading-order effects appear in the frequencies of any of these transitions. Thus, there is no leading-order $1 S-2 S$ spectroscopic signal for Lorentz or $C P T$ violation in either free $\mathrm{H}$ or free $\overline{\mathrm{H}}[22]$.

The dominant subleading energy-level shifts involving the $C P T$ - and Lorentz-breaking couplings in free $\mathrm{H}$ and $\overline{\mathrm{H}}$ arise as relativistic corrections of order $\alpha^{2}$. These differ for some of the $1 S$ and $2 S$ levels and therefore could, in principle, lead to observable effects. For example, the term proportional to $b_{3}^{e}$ in Eq. (1) produces a frequency shift in the $m_{F}=1 \rightarrow m_{F^{\prime}}=1$ line relative to the $m_{F}=$ $0 \rightarrow m_{F^{\prime}}=0$ line (which remains unshifted), given by $\delta \nu_{1 S-2 S}^{\mathrm{H}} \approx-\alpha^{2} b_{3}^{e} / 8 \pi$. Similarly, the proton-antiproton corrections are also suppressed by factors at least of order $\alpha^{2} \simeq 5 \times 10^{-5}$. The suppression factors reduce the signals in both free $\mathrm{H}$ and free $\overline{\mathrm{H}}$ to levels that could, in principle, be excluded by results from feasible $g-2$ experiments. In fact, the estimated attainable bound [16] on $b_{3}^{e}$ from electron-positron $g-2$ experiments performed with existing technology would suffice to place a bound of $\delta \nu_{1 S-2 S}^{\mathrm{H}} \lesssim 5 \mu \mathrm{Hz}$ on observable shifts of the $1 S-2 S$ frequency in free $\mathrm{H}$ from the electron-positron sector. This is below the resolution of the $1 S-2 S$ line center. Although no Penning-trap $g-2$ experiments on protons and antiprotons have yet been performed, bounds attainable in such experiments would also yield tighter constraints on the proton-antiproton parameters than would be obtained in $1 S-2 S$ measurements.

At first sight, it may seem surprising that bounds from $g-2$ experiments can constrain observable effects in comparisons of $1 S-2 S$ transitions in free $\mathrm{H}$ and $\overline{\mathrm{H}}$. The conventional figure of merit for $C P T$ breaking in $g-2$ experiments involving the difference of the electron and positron $g$ factors is $r_{g}=\left|g_{e^{-}}-g_{e^{+}}\right| / g_{\text {av }} \leqslant 2 \times 10^{-12}$ [23], which is 6 orders of magnitude weaker than the idealized resolution of the $1 S-2 S$ line, $\Delta \nu_{1 S-2 S} / \nu_{1 S-2 S} \simeq 10^{-18}$. However, the use of $r_{g}$ in Penning-trap $g-2$ experiments can be inappropriate in the present theoretical context [16]. The relevant physical issues are the absolute frequency resolution and the sensitivity to $C P T$ - and Lorentz-violating effects. The absolute frequency resolution in $g-2$ measurements is approximately $1 \mathrm{~Hz}$, which is about 3 orders of magnitude poorer than the idealized $1 S-2 S$ line-center resolution. In contrast, the $g-2$ experiments involve spin-flip transitions that induce direct sensitivity to $b_{3}^{e}$, whereas the $1 S-2 S$ transitions in free $\mathrm{H}$ or $\overline{\mathrm{H}}$ are sensitive only to the suppressed combination $\alpha^{2} b_{3}^{e} / 8 \pi$. The resulting bound on $b_{3}^{e}$ from $1 S-2 S$ comparisons is thus about 2 orders of magnitude weaker than that from electron-positron $g-2$ experiments. The above discussion suggests that experiments with $\mathrm{H}$ and $\overline{\mathrm{H}}$ might obtain tighter bounds by studying transitions between states with different spin configurations. Accomplishing this requires the presence of external fields.

We next consider spectroscopy of $\mathrm{H}$ or $\overline{\mathrm{H}}$ confined within a magnetic trap with an axial bias magnetic field, such as an Ioffe-Pritchard trap [24]. This situation is directly relevant to proposed experiments [9]. Denote each of the four $1 S$ and $2 S$ hyperfine Zeeman levels in order of increasing energy in a magnetic field $B$ with the labels $|a\rangle_{n}$, $|b\rangle_{n},|c\rangle_{n},|d\rangle_{n}$, with $n=1$ or 2 , for both $\mathrm{H}$ and $\overline{\mathrm{H}}$. For $\mathrm{H}$, the mixed-spin states are given in terms of the basis states $\left|m_{J}, m_{I}\right\rangle$ as

$$
\begin{aligned}
& |c\rangle_{n}=\sin \theta_{n}\left|-\frac{1}{2}, \frac{1}{2}\right\rangle+\cos \theta_{n}\left|\frac{1}{2},-\frac{1}{2}\right\rangle, \\
& |a\rangle_{n}=\cos \theta_{n}\left|-\frac{1}{2}, \frac{1}{2}\right\rangle-\sin \theta_{n}\left|\frac{1}{2},-\frac{1}{2}\right\rangle .
\end{aligned}
$$

The mixing angles $\theta_{n}$ depend on the principal quantum number $n$ and obey $\tan 2 \theta_{n} \approx(51 \mathrm{mT}) / n^{3} B$. Prior to excitation, the states that remain confined in the trap are the low-field seekers, $|c\rangle_{1}$ and $|d\rangle_{1}$. However, spinexchange collisions $|c\rangle_{1}+|c\rangle_{1} \rightarrow|b\rangle_{1}+|d\rangle_{1}$ lead to a loss of population of the $|c\rangle_{1}$ states over time, resulting in confinement of primarily $|d\rangle_{1}$ states.

Transitions between the unmixed-spin states $|d\rangle_{1}$ and $|d\rangle_{2}$ are field independent for small values of the magnetic field. It would therefore seem natural to compare the frequency $\nu_{d}^{\mathrm{H}}$ for the $1 S-2 S$ transition $|d\rangle_{1} \rightarrow|d\rangle_{2}$ in $\mathrm{H}$ with the frequency $\nu_{d}^{\overline{\mathrm{H}}}$ for the corresponding transition in $\overline{\mathrm{H}}$. However, since in $\mathrm{H}$ the spin configurations of the $|d\rangle_{1}$ and $|d\rangle_{2}$ states are the same, there are again no unsuppressed frequency shifts. The same result holds for $\overline{\mathrm{H}}$. Thus, to leading order, we find $\delta \nu_{d}^{\mathrm{H}}=\delta \nu_{d}^{\overline{\mathrm{H}}} \simeq 0$.

A theoretically interesting alternative would be to consider instead the $1 S-2 S$ transition $|c\rangle_{1} \rightarrow|c\rangle_{2}$ in $\mathrm{H}$ and the corresponding transition in $\overline{\mathrm{H}}$. The idea would be to take advantage of the mixed nature of these states in a nonzero magnetic field. The $n$ dependence in the hyperfine splitting produces a spin-mixing difference between the $1 S$ and 
$2 S$ levels, giving an unsuppressed frequency shift in $1 S-2 S$ transitions between the $|c\rangle_{1}$ and $|c\rangle_{2}$ states:

$$
\begin{aligned}
\delta \nu_{c}^{\mathrm{H}} \approx-\kappa\left(b_{3}^{e}-b_{3}^{p}-d_{30}^{e} m_{e}\right. & \\
& \left.+d_{30}^{p} m_{p}-H_{12}^{e}+H_{12}^{p}\right) / 2 \pi,
\end{aligned}
$$

where $\kappa \equiv \cos 2 \theta_{2}-\cos 2 \theta_{1}$. The analogous $1 S$ - $2 S$ frequency shift $\delta \nu_{c}^{\overline{\mathrm{H}}}$ for $\overline{\mathrm{H}}$ in the same magnetic field can also be found. The hyperfine states in $\overline{\mathrm{H}}$ have opposite positron and antiproton spin assignments relative to those of the electron and proton in $\mathrm{H}$, so $\delta \nu_{c}^{\overline{\mathrm{H}}}$ is given by an expression identical to that for $\delta \nu_{c}^{\mathrm{H}}$ in Eq. (4) except that the signs of $b_{3}^{e}$ and $b_{3}^{p}$ are changed. The frequencies $\nu_{c}^{\mathrm{H}}$ and $\nu_{c}^{\overline{\mathrm{H}}}$ depend on spatial components of Lorentz-violating couplings and so would exhibit diurnal variations in the comoving Earth frame. There would also be a nonzero instantaneous difference $\Delta \nu_{1 S-2 S, c} \equiv$ $\nu_{c}^{\mathrm{H}}-\nu_{c}^{\overline{\mathrm{H}}} \approx-\kappa\left(b_{3}^{e}-b_{3}^{p}\right) / \pi$ for measurements made in the same magnetic trapping fields. The value of this difference would depend on the $1 S-2 S$ spin-mixing difference controlled by $\kappa$ [25].

The theoretical gain in sensitivity to $C P T$ and Lorentz violation of the $|c\rangle_{1} \rightarrow|c\rangle_{2}$ transition relative to that of $|d\rangle_{1} \rightarrow|d\rangle_{2}$ would be of order $4 / \alpha^{2} \simeq 10^{5}$. However, since the $1 S-2 S$ transition $|c\rangle_{1} \rightarrow|c\rangle_{2}$ in $\mathrm{H}$ and $\overline{\mathrm{H}}$ is field dependent, any experiment would need to overcome Zeeman broadening due to the inhomogeneous trapping fields. For example, at $B \simeq 10 \mathrm{mT}$ the $1 S-2 S$ linewidth for the $|c\rangle_{1} \rightarrow|c\rangle_{2}$ transition is broadened to over $1 \mathrm{MHz}$ for both $\mathrm{H}$ and $\overline{\mathrm{H}}$ even at a temperature of $100 \mu \mathrm{K}$. Existing techniques might partially mitigate this effect, but the development of other experimental methods would appear necessary to attain resolutions on the order of the natural linewidth.

As an alternative to optical spectroscopy of the $1 S-2 S$ line, we consider frequency measurements of transitions in the hyperfine Zeeman effect. Since transitions between $F=0$ and $F^{\prime}=1$ hyperfine states have been measured with accuracies better than $1 \mathrm{mHz}$ in a hydrogen maser [26], hyperfine transitions in masers and in trapped $\mathrm{H}$ and $\overline{\mathrm{H}}$ are interesting candidates for tests of $C P T$ and Lorentz symmetry.

In the $1 S$ ground state of hydrogen, all four hyperfine levels acquire energy shifts due to $C P T$ - and Lorentzviolating effects. Each energy shift contains an identical contribution $a_{0}^{e}+a_{0}^{p}-c_{00}^{e} m_{e}-c_{00}^{p} m_{p}$ that leaves transition frequencies unaffected. The remaining spindependent contributions to the energy shifts are

$$
\begin{aligned}
& \Delta E_{a}^{\mathrm{H}} \simeq \hat{\kappa}\left(b_{3}^{e}-b_{3}^{p}-d_{30}^{e} m_{e}+d_{30}^{p} m_{p}-H_{12}^{e}+H_{12}^{p}\right), \\
& \Delta E_{b}^{\mathrm{H}} \simeq b_{3}^{e}+b_{3}^{p}-d_{30}^{e} m_{e}-d_{30}^{p} m_{p}-H_{12}^{e}-H_{12}^{p}, \\
& \Delta E_{c}^{\mathrm{H}} \simeq-\Delta E_{a}^{\mathrm{H}}, \quad \Delta E_{d}^{\mathrm{H}} \simeq-\Delta E_{b}^{\mathrm{H}},
\end{aligned}
$$

where $\hat{\kappa} \equiv \cos 2 \theta_{1}$. In zero magnetic field $\hat{\kappa}=0$, so the energies of $|a\rangle_{1}$ and $|c\rangle_{1}$ are unshifted. However, $|b\rangle_{1}$ and $|d\rangle_{1}$ acquire equal and opposite energy shifts.
The degeneracy of the three $F=1$ ground-state hyperfine levels is therefore lifted even for $B=0$ [27]. For example, the transitions $|d\rangle_{1} \rightarrow|a\rangle_{1}$ and $|b\rangle_{1} \rightarrow|a\rangle_{1}$ exhibit an unsuppressed and diurnally varying frequency difference $\left|\Delta \nu_{d-b}^{\mathrm{H}}\right| \approx \mid b_{3}^{e}+b_{3}^{p}-d_{30}^{e} m_{e}-d_{30}^{p} m_{p}-$ $H_{12}^{e}-H_{12}^{p} \mid / \pi$. With nonzero values of the magnetic field, all four hyperfine Zeeman levels acquire energy shifts. For $|a\rangle_{1}$ and $|c\rangle_{1}$, they are controlled by the spin-mixing parameter $\hat{\kappa}$, increasing with $B$ and attaining $\hat{\kappa} \simeq 1$ when $B \simeq 0.3 \mathrm{~T}$.

The conventional $\mathrm{H}$ maser operates on the fieldindependent $\sigma$ transition $|c\rangle_{1} \rightarrow|a\rangle_{1}$ in the presence of a small $\left(B \lesssim 10^{-6} \mathrm{~T}\right)$ magnetic field. Since $\hat{\kappa} \lesssim 10^{-4}$ in this case, the leading-order effects due to $C P T$ and Lorentz violation in high-precision measurements of the maser line $|c\rangle_{1} \rightarrow|a\rangle_{1}$ are suppressed. However, the frequency difference between the field-dependent transitions $|d\rangle_{1} \rightarrow|a\rangle_{1}$ and $|b\rangle_{1} \rightarrow|a\rangle_{1}$ is shifted relative to its usual value by $\Delta \nu_{d-b}^{\mathrm{H}}$, and the associated diurnal variations would provide an unsuppressed signal of $C P T$ and Lorentz violation. Although measurements of this difference with existing techniques are possible in principle, the frequency resolution would be significantly less than that of the field-independent $\sigma$ line because of broadening due to field inhomogeneities. Moreover, an unambiguous resolution of this signal would require distinguishing it from possible backgrounds arising from residual Zeeman splittings.

The issue of background splittings could, in principle, be addressed by direct comparison of transitions between hyperfine Zeeman levels in $\mathrm{H}$ and $\overline{\mathrm{H}}$. Furthermore, the frequency dependence on the magnetic field could be eliminated to first order by using a field-independent transition point. One possibility might be to perform highresolution radio frequency spectroscopy on the $|d\rangle_{1} \rightarrow|c\rangle_{1}$ transition in trapped $\mathrm{H}$ and $\overline{\mathrm{H}}$ at the field-independent transition point $B \simeq 0.65 \mathrm{~T}$. Among the experimental issues to consider would be Doppler broadening and that the relatively high bias field implies potentially larger field inhomogeneities, so cooling to temperatures of $100 \mu \mathrm{K}$ with a good signal-to-noise ratio and a stiff box shape for the trapping potential may be needed to obtain frequency resolutions of order $1 \mathrm{mHz}$.

At this bias-field strength, the electron and proton spins in the state $|c\rangle_{1}$ are highly polarized with $m_{J}=\frac{1}{2}$ and $m_{I}=-\frac{1}{2}$. The transition $|d\rangle_{1} \rightarrow|c\rangle_{1}$ is effectively a proton spin-flip transition. We find the frequency shifts for $\mathrm{H}$ and $\overline{\mathrm{H}}$ are $\delta \nu_{c \rightarrow d}^{\mathrm{H}} \approx\left(-b_{3}^{p}+d_{30}^{p} m_{p}+H_{12}^{p}\right) / \pi$ and $\delta \nu_{c \rightarrow d}^{\overline{\mathrm{H}}} \approx\left(b_{3}^{p}+d_{30}^{p} m_{p}+H_{12}^{p}\right) / \pi$. The frequencies $\nu_{c \rightarrow d}^{\mathrm{H}}$ and $\nu_{c \rightarrow d}^{\overline{\mathrm{H}}}$ would exhibit diurnal variations. Their instantaneous difference,

$$
\Delta \nu_{c \rightarrow d} \equiv \nu_{c \rightarrow d}^{\mathrm{H}}-\nu_{c \rightarrow d}^{\overline{\mathrm{H}}} \approx-2 b_{3}^{p} / \pi,
$$

could provide a direct, clean, and accurate test of $C P T$ violating couplings $b_{3}^{p}$ for the proton [28]. 
Relevant figures of merit for the various direct and diurnal-variation signals described in this paper can be introduced in analogy with those for Penning-trap tests [16]. As one example, a possible figure of merit for the signal in Eq. (6) would be

$$
\begin{aligned}
r_{r f, c \rightarrow d}^{\mathrm{H}} & \equiv\left|\left(\mathcal{E}_{1, d}^{\mathrm{H}}-\mathcal{E}_{1, c}^{\mathrm{H}}\right)-\left(\mathcal{E}_{1, d}^{\overline{\mathrm{H}}}-\mathcal{E}_{1, c}^{\overline{\mathrm{H}}}\right)\right| / \mathcal{E}_{1, \mathrm{av}}^{\mathrm{H}} \\
& \approx 2 \pi\left|\Delta \nu_{c \rightarrow d}\right| / m_{\mathrm{H}},
\end{aligned}
$$

where $\mathcal{E}_{1, d}^{\mathrm{H}}, \mathcal{F}_{1, c}^{\mathrm{H}}$ and the corresponding quantities for $\overline{\mathrm{H}}$ each denote a relativistic energy in a ground-state hyperfine level. The mass $m_{\mathrm{H}}$ is the atomic mass of $\mathrm{H}$. Thus, for example, attaining a frequency resolution of about $1 \mathrm{mHz}$ corresponds to an estimated upper bound of $r_{r f, c \rightarrow d}^{\mathrm{H}} \lesssim$ $5 \times 10^{-27}$. The limit on the $C P T$ - and Lorentz-violating coupling $b_{3}^{p}$ would then be $\left|b_{3}^{p}\right| \lesssim 10^{-18} \mathrm{eV}$, which is about 3 orders of magnitude better than estimated attainable bounds [16] from $g-2$ experiments in Penning traps and over 4 orders of magnitude better than bounds attainable from $1 S-2 S$ transitions [29].

In summary, we have shown that spin-mixed $1 S-2 S$ and spin-flip hyperfine spectroscopic signals for Lorentz and $C P T$ violation appear in $\mathrm{H}$ or $\overline{\mathrm{H}}$ atoms confined in a magnetic trap. These signals are unsuppressed by any power of the fine-structure constant and would represent observable consequences of qualitatively new physics originating at the Planck scale.

This work is supported in part by the U.S. DOE under Grant No. DE-FG02-91ER40661 and by the NSF under Grant No. PHY-9503756.

[1] See, for example, The Hydrogen Atom, edited by G.F. Bassani, M. Inguscio, and T. W. Hänsch (Springer-Verlag, Berlin, 1989).

[2] G. Baur et al., Phys. Lett. B 368, 251 (1996).

[3] G. Blanford et al., Phys. Rev. Lett. 80, 3037 (1998).

[4] See, for example, M. Charlton et al., Phys. Rep. 241, 65 (1994); Antihydrogen, edited by J. Eades (J. C. Baltzer, Geneva, 1993).

[5] See, for example, T.W. Hänsch, in Atomic Physics 14, edited by D. J. Wineland, C.E. Wieman, and S. J. Smith, AIP Conf. Proc. No. 323 (AIP, New York, 1995).

[6] T. Udem et al., Phys. Rev. Lett. 79, 2646 (1997).

[7] C. L. Cesar et al., Phys. Rev. Lett. 77, 255 (1996).

[8] G. Blanford et al., Phys. Rev. D 57, 6649 (1998).

[9] B. Brown et al., Nucl. Phys. (Proc. Suppl.) A56, 326 (1997); M. H. Holzscheiter et al., ibid. A56, 336 (1997).

[10] The discrete symmetries $C, P, T$ are discussed, for example, in R.G. Sachs, The Physics of Time Reversal (University of Chicago Press, Chicago, 1987).

[11] V.A. Kostelecký and S. Samuel, Phys. Rev. Lett. 63, 224 (1989); ibid. 66, 1811 (1991); Phys. Rev. D 39, 683 (1989); ibid. 40, 1886 (1989); V. A. Kostelecký and R. Potting, Nucl. Phys. B359, 545 (1991); Phys. Lett. B 381, 89 (1996).

[12] D. Colladay and V. A. Kostelecký, Phys. Rev. D 55, 6760 (1997); ibid. 58, 116002 (1998).

[13] V.A. Kostelecký and R. Potting, in Gamma RayNeutrino Cosmology and Planck Scale Physics, edited by
D. B. Cline (World Scientific, Singapore, 1993) (hep-th/ 9211116); Phys. Rev. D 51, 3923 (1995); D. Colladay and V. A. Kostelecký, Phys. Lett. B 344, 259 (1995); Phys. Rev. D 52, 6224 (1995); V. A. Kostelecký and R. Van Kooten, Phys. Rev. D 54, 5585 (1996).

[14] OPAL Collaboration, R. Ackerstaff et al., Z. Phys. C 76, 401 (1997); DELPHI Collaboration, M. Feindt et al., Report No. DELPHI 97-98 CONF 80, 1997.

[15] V. A. Kostelecký, Phys. Rev. Lett. 80, 1818 (1998).

[16] R. Bluhm, V.A. Kostelecký, and N. Russell, Phys. Rev. Lett. 79, 1432 (1997); Phys. Rev. D 57, 3932 (1998).

[17] O. Bertolami et al., Phys. Lett. B 395, 178 (1997).

[18] In experiments with $\mathrm{H}$ and $\overline{\mathrm{H}}$, suitable field redefinitions can eliminate various combinations of these couplings, which thus must be unobservable [12]. To show this explicitly, we keep all couplings in what follows.

[19] See, for example, G. Breit, Phys. Rev. 34, 553 (1929).

[20] Note that comparisons of results from different experiments may necessitate inclusion of geometrical factors associated with differing spin-quantization axes.

[21] B. Cagnac, G. Grynberg, and F. Biraben, J. Phys. 34, 845 (1973).

[22] This result is consistent with the demonstration in Ref. [16] that observable $C P T$-violating effects must also involve $C T$ violation and spin-flip processes.

[23] See, for example, R. M. Barnett et al., Phys. Rev. D 54, 1 (1996).

[24] Y.V. Gott, M. S. Ioffe, and V.G. Tel'kovskii, Nucl. Fusion (Suppl.) 3, 1045 (1962); D. E. Pritchard, Phys. Rev. Lett. 51, 1336 (1983).

[25] Theoretically optimal situations would have maximal $\kappa$, which occurs at $B \simeq 0.01 \mathrm{~T}$ when $\kappa \simeq 0.67$.

[26] N. F. Ramsey, Phys. Scr. T59, 323 (1995).

[27] The removal of the $|b\rangle-|d\rangle$ degeneracy at zero field is compatible with Kramer's theorem since the Lorentzviolating coefficients in Eq. (5) also violate $T$. This splitting might be experimentally detected by seeking quantum beats at the difference frequency.

[28] All experiments described here are sensitive only to spatial components of $C P T$-violating couplings. For sensitivity to purely timelike components such as $b_{0}^{e}$, a boost would be required. This can enhance $C P T$ - and Lorentzviolating effects [15]. Although the proposed experiments [8] to measure the fine structure and Lamb shift with a relativistic beam of $\overline{\mathrm{H}}$ are expected to have poorer resolutions than the others discussed above, bounds on $b_{0}^{e}$ and $b_{0}^{p}$ may be attainable.

[29] In comparison, note that the frequency resolution of the most accurate clock-comparison experiments, which also constrain certain types of Lorentz violation, lies below $1 \mu \mathrm{Hz}$ [30]. However, the theoretical interpretation of these experiments is more difficult because relatively complex nuclei are involved, and, in any event, no leading-order bounds are obtained on $b_{3}^{p}$ in isolation from other couplings [31].

[30] V.W. Hughes, H. G. Robinson, and V. Beltran-Lopez, Phys. Rev. Lett. 4, 342 (1960); R. W. P. Drever, Philos. Mag. 6, 683 (1961); J.D. Prestage et al., Phys. Rev. Lett. 54, 2387 (1985); S. K. Lamoreaux et al., ibid. 57, 3125 (1986); T.E. Chupp et al., ibid. 63, 1541 (1989); C. J. Berglund et al., ibid. 75, 1879 (1995).

[31] V. A. Kostelecký and C. D. Lane (to be published). 